\title{
Coherent Optical Control of the Ultrafast Dephasing of Phonon-Plasmon Coupling in a Polar Semiconductor Using a Pulse Train of Below-Band-Gap Excitation
}

\author{
J. D. Lee ${ }^{1}$ and Muneaki Hase $\mathrm{H}^{2,3}$ \\ ${ }^{1}$ School of Materials Science, Japan Advanced Institute of Science and Technology, Ishikawa 923-1292, Japan \\ ${ }^{2}$ Institute of Applied Physics, University of Tsukuba, 1-1-1 Tennodai, Tsukuba 305-8573, Japan \\ ${ }^{3}$ PRESTO, Japan Science and Technology Agency, 4-1-8 Honcho, Kawaguchi 332-0012, Japan
}

(Received 23 June 2008; published 4 December 2008)

\begin{abstract}
In the investigation of the nonequilibrium ultrafast dynamics of the coherent phonon-plasmon coupled modes in a polar semiconductor, we predict theoretically that their coherent oscillations can be efficiently controlled by using the pulse train of below-band-gap excitation. The dynamics of the coherent modes are driven by the virtual electron-hole pairs, which would avoid dephasing sources such as accumulation of photoexcited charges and spontaneous emission. This implies that carrier mobility can also be efficiently controlled and dramatically enhanced by synchronizing the pulse train with the coherent oscillation of the carrier-relevant coupled mode.
\end{abstract}

PACS numbers: 63.20.kd, 42.65.Re, 72.20.Jv, 78.66.Fd

Below-band-gap excitation of semiconductors suggests a key technique to realize the dephasing-free dynamics, such as efficient coherent control of spins [1] and extremely stable solitons [2]. The ac Stark effect shows that virtual excitations by the below-band-gap excitation give rise to exactly same physical processes as real ones except that they cause no relaxation and have no lifetime [3-5].

The carrier mobility is one of the central quantities in the operation of modern semiconductor devices, such as high electron mobility transistors (HEMT), field effect transistors (FET), or in the generation and manipulation of terahertz (THz) radiation from semiconductor surfaces by the photo-Dember effect [6]. The carrier mobility $\mu$ is given by $\mu=e \bar{\tau} / m^{*}$, where $e$ is the electron charge, $m^{*}$ the carrier effective mass, and $\bar{\tau}$ the relaxation time. $\bar{\tau}$ could be obtained by $\mathrm{THz}$ time-domain spectroscopy [7], but the accessible carrier density was limited to one lower than $\mathcal{O}\left(10^{16}\right) \mathrm{cm}^{-3}$ because of the non-Drude behavior at higher carrier densities. In contrast, Raman investigation of $\mathrm{SiC}$ polytypes has shown that the linewidth of the carrier-relevant LO phonon-plasmon coupled (LOPC) modes [8] can be directly matched to the carrier mobility obtained from Hall measurements in the high carrier density region [15]. Moreover, Hase has recently measured the relaxation time $\bar{\tau}_{L+}$ of the coherent carrier-relevant mode (i.e., $L+$ mode) from its real-time relaxation using timeresolved electro-optic detection and time-frequency analysis in GaAs [16], showing that $\mu \approx e \bar{\tau}_{L+} / m^{*}$ could give a good estimation of carrier mobility.

In this Letter, we propose a new theory for controlling the coherent motion of the LOPC mode and eventually the carrier mobility $\mu$. In the proposition, for instance, in $\mathrm{GaAs}$, the virtual excitations by the below-band-gap excitation would screen the space-charge field in the surface region and drive the coherent modes just like real ones. The virtual carriers have no lifetime, so that they would not cause undesirable scattering events with the coherent modes or other (virtual) carriers by succeeding pulses. This makes the virtual excitation preserve the coherence by suppressing the dephasing sources. In particular, it is found that carrier mobility can be dramatically enhanced by synchronizing the pulse train of below-band-gap excitation with the coherent oscillation of the carrier-relevant mode.

We consider the creation of continuum carriers (electron-hole pairs) by laser irradiation, which excites phonons and plasmons in a polar semiconductor. Phonons and plasmons interact with each other through an explicit phonon-plasmon coupling $g$. The resulting Hamiltonian $\mathcal{H}\left(=\mathcal{H}_{0}+\mathcal{V}\right)$ reads

$$
\begin{aligned}
\mathcal{H}_{0}= & \sum_{\mathbf{k}} \epsilon_{\mathbf{k}}^{v} d_{\mathbf{k}}^{\dagger} d_{\mathbf{k}}+\sum_{\mathbf{k}} \epsilon_{\mathbf{k}}^{c} c_{\mathbf{k}}^{\dagger} c_{\mathbf{k}}+\sum_{\mathbf{q}} \omega_{\mathrm{LO}} a_{\mathbf{q}}^{\dagger} a_{\mathbf{q}} \\
& +\sum_{\mathbf{k}} \sum_{\mathbf{q}} M_{\mathbf{q}} c_{\mathbf{k}}^{\dagger} c_{\mathbf{k}+\mathbf{q}}\left(a_{-\mathbf{q}}+a_{\mathbf{q}}^{\dagger}\right)+\sum_{\mathbf{q}} \omega_{\mathrm{PL}} b_{\mathbf{q}}^{\dagger} b_{\mathbf{q}} \\
& +\sum_{\mathbf{k}} \sum_{\mathbf{q}} V_{\mathbf{q}} c_{\mathbf{k}}^{\dagger} c_{\mathbf{k}+\mathbf{q}}\left(b_{-\mathbf{q}}+b_{\mathbf{q}}^{\dagger}\right) \\
& +g \sum_{\mathbf{q}}\left(a_{\mathbf{q}}+a_{-\mathbf{q}}^{\dagger}\right)\left(b_{-\mathbf{q}}+b_{\mathbf{q}}^{\dagger}\right), \\
\mathcal{V}= & \frac{1}{2} \sum_{\mathbf{k}} \Omega_{0} e^{i \omega \tau} \bar{\Theta}(\tau) c_{\mathbf{k}}^{\dagger} d_{-\mathbf{k}}^{\dagger}+\text { H.c. }
\end{aligned}
$$

$d_{\mathbf{k}}^{\dagger}\left(d_{\mathbf{k}}\right)$ and $c_{\mathbf{k}}^{\dagger}\left(c_{\mathbf{k}}\right)$ are the operators of the photoexcited hole and electron, respectively. $a_{\mathbf{q}}^{\dagger}\left(a_{\mathbf{q}}\right)$ is the operator for the LO phonon $\omega_{\mathrm{LO}}$, while $b_{\mathbf{q}}^{\dagger}\left(b_{\mathbf{q}}\right)$ for the plasmon $\omega_{\mathrm{PL}}$. The plasmon bath describes the degenerate sea of doped carriers, which is made possible by the quasiboson approximation [17]. $\omega_{\mathrm{PL}}$ is determined by $\sqrt{4 \pi n e^{2} / \varepsilon_{\infty} m^{*}}$ with the carrier density $n$. The photoexcited electron interacts with the LO phonon via the Fröhlich coupling $M_{\mathbf{q}}=\sqrt{2 \pi \omega_{\mathrm{LO}}}\left(1 / \varepsilon_{\infty}-1 / \varepsilon_{0}\right)^{1 / 2} /|\mathbf{q}|$ and with the plas- 
mon via the Lundqvist form $V_{\mathbf{q}}=\sqrt{2 \pi \omega_{\mathrm{PL}}}\left(1 / \varepsilon_{\infty}\right) /|\mathbf{q}|$ [18], where $\varepsilon_{0}$ and $\varepsilon_{\infty}$ are low- and high-frequency dielectric constants. $\mathcal{V}$ is the light-matter interaction by the pumping pulse train. The strength of the light-matter coupling is quantified by $\Omega_{0}\left(=d_{c v} A\right)$, where $d_{c v}$ is the dipole matrix and $A$ is the field amplitude of the laser. Putting the length of a single pulse as $\sigma$ and the interval between pulses as $\Delta(\Delta \gg \sigma)$, we note $\bar{\Theta}(\tau)=\sum_{n=0}^{n=N-1}[\Theta(\tau+$ $n \Delta)-\Theta(\tau+n \Delta-\sigma)]$ for a pulse train with $N$ pulses, where $\Theta(\tau)$ is the Heaviside step function. $\omega$ is the pulse energy.

We define $\delta$ ( $\equiv \epsilon_{\mathrm{G}}-\omega ; \epsilon_{\mathrm{G}}$ : energy gap) as the detuning of the laser with respect to the electron-hole continuum, so that $\delta>0$ signifies the below-band-gap excitation. An application of the below-band-gap excitation pulses introduces the virtual electron-hole continuum in a semiconductor, which excites phonons and plasmons. It is a pure quantum mechanical feature, that is sharply in contrast with the classical description [19]. The manipulation of coherent phonons and plasmons using the virtual electron-hole pair avoids the dephasing processes. First, it does not accumulate the photoexcited carriers in a conduction band as shown in Fig. 1. Otherwise, there would be significant damping of coherent phonon and plasmon due to scattering with optically generated electrons and holes [20]. Second, no change of the carrier density suppresses the free-streaming current of the counter-directed carrier stream by succeeding pulses [21]. Third, when a semiconductor is illuminated by a laser with an electric field $E_{\alpha}$, the field energy $\mathcal{E}$ stored in the semiconductor is $\mathcal{E}=$ $-\sum_{\alpha \beta} \chi_{\alpha \beta}(\omega) E_{\alpha} E_{\beta}$ with the optical response function $\chi_{\alpha \beta}(\omega)$. For $\omega<\epsilon_{\mathrm{G}}$, the dissipative part $\operatorname{Im}\left[\chi_{\alpha \beta}(\omega)\right]$ would be zero, so that the spontaneous emission is suppressed [1].

For a theoretical description of the nonequilibrium ultrafast dynamics of a semiconductor, it is a powerful idea to solve the time-dependent Schrödinger equation in the many-body Hilbert space spanning the whole system [22]. Because the total carrier density does not change, the dynamics is described by a single quantum state $|\Psi(\tau)\rangle$
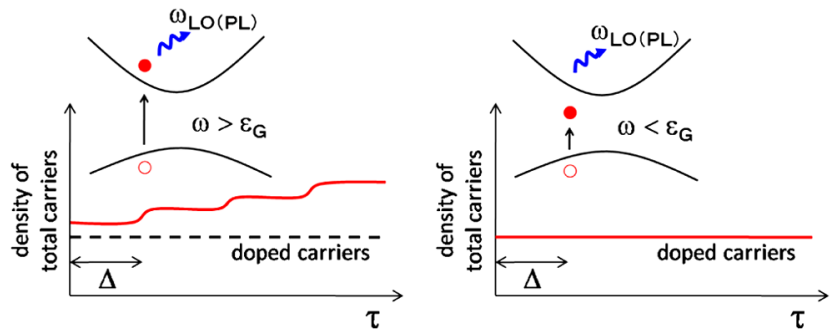

FIG. 1 (color online). Excitation of real (left) and virtual (right) electron-hole pair continuum. In contrast to the aboveband-gap excitation case, the below-band-gap excitation case $\left(\omega<\epsilon_{\mathrm{G}}\right)$ does not accumulate carriers (i.e., no photoexcited carrier). with $A \rightarrow 0$ (i.e., $\Omega_{0} \rightarrow 0$ )

$$
\begin{aligned}
|\Psi(\tau)\rangle= & C(\tau)|0\rangle_{c}|0\rangle_{v}|0\rangle_{\mathrm{LO}}|0\rangle_{\mathrm{PL}} \\
& +\sum_{\mathbf{q}} C_{\mathbf{q}}^{\mathrm{LO}}(\tau)|0\rangle_{c}|0\rangle_{v}|\mathbf{q}\rangle_{\mathrm{LO}}|0\rangle_{\mathrm{PL}} \\
& +\sum_{\mathbf{q}} C_{\mathbf{q}}^{\mathrm{PL}}(\tau)|0\rangle_{c}|0\rangle_{v}|0\rangle_{\mathrm{LO}}|\mathbf{q}\rangle_{\mathrm{PL}} \\
& +\sum_{\mathbf{k} \mathbf{k}^{\prime}} C_{\mathbf{k} \mathbf{k}^{\prime}}(\tau)|\mathbf{k}\rangle_{c}\left|\mathbf{k}^{\prime}\right\rangle_{v}|0\rangle_{\mathrm{LO}}|0\rangle_{\mathrm{PL}} \\
& +\sum_{\mathbf{k} \mathbf{k}^{\prime}} \sum_{\mathbf{q}} C_{\mathbf{k k}^{\prime} \mathbf{q}}^{\mathrm{LO}}(\tau)|\mathbf{k}\rangle_{c}\left|\mathbf{k}^{\prime}\right\rangle_{v}|\mathbf{q}\rangle_{\mathrm{LO}}|0\rangle_{\mathrm{PL}} \\
& +\sum_{\mathbf{k} \mathbf{k}^{\prime}} \sum_{\mathbf{q}} C_{\mathbf{k k}^{\prime} \mathbf{q}}^{\mathrm{PL}}(\tau)|\mathbf{k}\rangle_{c}\left|\mathbf{k}^{\prime}\right\rangle_{v}|0\rangle_{\mathrm{LO}}|\mathbf{q}\rangle_{\mathrm{PL}} .
\end{aligned}
$$

For $|\Psi(\tau)\rangle$, we restricted the many-body Hilbert space to contain just a single coherent phonon or plasmon. In $|\Psi(\tau)\rangle$, we denote $|\mathbf{k}\rangle_{c}=c_{\mathbf{k}}^{\dagger}|0\rangle_{c},|\mathbf{k}\rangle_{v}=d_{\mathbf{k}}^{\dagger}|0\rangle_{v},|\mathbf{q}\rangle_{\mathrm{LO}}=$ $a_{\mathbf{q}}^{\dagger}|0\rangle_{\mathrm{LO}}$, and $|\mathbf{q}\rangle_{\mathrm{PL}}=b_{\mathbf{q}}^{\dagger}|0\rangle_{\mathrm{PL}}$ for single particle states. The initial state $|\Psi(0)\rangle$ should be the ground state, i.e., $|\Psi(0)\rangle=|0\rangle_{c}|0\rangle_{v}|0\rangle_{\mathrm{LO}}|0\rangle_{\mathrm{PL}}$. The time-dependent Schrödinger equation $i \partial / \partial \tau|\Psi(\tau)\rangle=\mathcal{H}|\Psi(\tau)\rangle$ results in infinitely coupled differential equations among coefficients. The transient reflectivity change $\Delta R / R$ is the actual experimental attainment from electro-optic detection combined with a standard pump-probe method [16]. It would phenomenologically give $\Delta R / R \propto r_{L} P_{L}+r_{E} P_{E}$ [19], where $P_{L}$ and $P_{E}$ are the lattice and electronic polarizations, respectively, and $r_{L}$ and $r_{E}$ are their weight coefficients. From the explicit solution, we can calculate the transient polarizations from $P_{L}(\tau)=\langle\Psi(\tau)| \sum_{\mathbf{q}}\left[a_{\mathbf{q}}+\right.$ $\left.a_{\mathbf{q}}^{\dagger}\right]|\Psi(\tau)\rangle$ and $P_{E}(\tau)=\left\langle\Psi(\tau)\left|\sum_{\mathbf{q}}\left[b_{\mathbf{q}}+b_{\mathbf{q}}^{\dagger}\right]\right| \Psi(\tau)\right\rangle$. When we define $\mathcal{P}_{L}(\tau)$ and $\mathcal{P}_{E}(\tau)$ as

$$
\begin{aligned}
& \mathcal{P}_{L}(\tau)=\sum_{\mathbf{q}} C^{*}(\tau) C_{\mathbf{q}}^{\mathrm{LO}}(\tau)+\sum_{\mathbf{k} \mathbf{k}^{\prime} \mathbf{q}} C_{\mathbf{k} \mathbf{k}^{\prime}}^{*}(\tau) C_{\mathbf{k} \mathbf{k}^{\prime} \mathbf{q}}^{\mathrm{LO}}(\tau), \\
& \mathcal{P}_{E}(\tau)=\sum_{\mathbf{q}} C^{*}(\tau) C_{\mathbf{q}}^{\mathrm{PL}}(\tau)+\sum_{\mathbf{k} \mathbf{k}^{\prime} \mathbf{q}} C_{\mathbf{k} \mathbf{k}^{\prime}}^{*}(\tau) C_{\mathbf{k} \mathbf{k}^{\prime} \mathbf{q}}^{\mathrm{PL}}(\tau),
\end{aligned}
$$

we have $P_{L}(\tau)=2 \operatorname{Re}\left[\mathcal{P}_{L}(\tau)\right]$ and $P_{E}(\tau)=2 \operatorname{Re}\left[\mathcal{P}_{E}(\tau)\right]$.

As implied in $\mathcal{H}$, we consider the $n$-type GaAs. It is expected that the hole would not affect the relevant dynamics much. Therefore, we impose an approximation of an infinitely massive hole by simply taking a value of the energy gap $\epsilon_{G}$ for $\epsilon_{\mathrm{k}}^{v}$, i.e., $\epsilon_{\mathrm{k}}^{v}=\epsilon_{\mathrm{G}}=1.4 \mathrm{eV}$. In addition, we note $\omega_{\mathrm{LO}}=8.8 \mathrm{THz}, \epsilon_{\mathbf{k}}^{c}=\mathbf{k}^{2} / 2 m^{*}\left(m^{*}=0.07\right)$, $\varepsilon_{\infty}=11.3$, and $\varepsilon_{0}=13.3$. In order to incorporate the free relaxation of the phonon and plasmon without any external control [23], we simply replace $\omega_{\mathrm{LO}} \rightarrow \omega_{\mathrm{LO}}+$ $i \gamma_{\mathrm{LO}}$ and $\omega_{\mathrm{PL}} \rightarrow \omega_{\mathrm{PL}}+i \gamma_{\mathrm{PL}}$. We define the relaxation times $\bar{\tau}_{\mathrm{LO}} \equiv 1 / \gamma_{\mathrm{LO}}$ and $\bar{\tau}_{\mathrm{PL}} \equiv 1 / \gamma_{\mathrm{PL}} \cdot \bar{\tau}_{\mathrm{LO}} \gg \bar{\tau}_{\mathrm{PL}}$ is typically expected and $\bar{\tau}_{\mathrm{LO}}=2.5 \mathrm{ps}$ and $\bar{\tau}_{\mathrm{PL}}=150 \mathrm{fs}$ are fixed in our investigation [24]. In the density range of our interest, $n>n_{0} \quad\left(n_{0}=0.75 \times 10^{18} \mathrm{~cm}^{-3}\right.$ giving 
$\left.\omega_{\mathrm{PL}}=\omega_{\mathrm{LO}}\right)$, the density dependence of $\gamma_{\mathrm{PL}}$ is tiny [25]. We fix the length of a single pulse as $\sigma=10 \mathrm{fs}$.

According to Fig. 2(a), the coupling between the phonon and plasmon results in new eigenmodes, the carrierrelevant $L+$ mode and the lattice-relevant $L-$ mode [8], whose energies are given by $\omega_{L_{ \pm}}=\frac{1}{2}\left(\omega_{\mathrm{PL}}+\omega_{\mathrm{LO}}\right) \pm \frac{1}{2} \times$ $\sqrt{\left(\omega_{\mathrm{PL}}-\omega_{\mathrm{LO}}\right)^{2}+4 g^{2}}$. In Fig. 2(b), transient electrooptical responses $\Delta R / R$ are provided as $\Delta R / R \propto P_{L}(\tau)+$ $P_{E}(\tau)$ by simply taking $r_{L} / r_{E}=1$. In the frequency-time space of Fig. 2(c), it is clear that the oscillations of $\Delta R / R$ are composed of two modes: i.e., the $L+$ (higher frequency) and $L-$ (lower frequency) mode. Most interestingly, the pulse train with $N=4$ is found to appreciably sustain the coherent oscillation of $L+$ mode compared to a single pulse $(N=1)$.

Our main interest is to investigate how to control the coherent motion of the $L+$ mode, because it is directly connected to the carrier mobility of a semiconductor through $\mu \approx e \bar{\tau}_{L+} / m^{*}$ [16], where $\bar{\tau}_{L+}$ is the relaxation time of the $L+$ mode. In Fig. 3, adopting the $N=8$ pulse train, we display the coherent motion of both $L+$ and $L-$ modes with respect to two controllable parameters: one is the carrier density $n$, and the other is the pulse interval $\Delta$. In the left panel of Fig. 3, the carrier density $n$ is changed from $1.5 \times 10^{18} \mathrm{~cm}^{-3}$ to $3 \times 10^{18} \mathrm{~cm}^{-3}$ for the pulse interval $\Delta=2 \pi / \omega_{L+}$. The frequency of the $L+$ mode shifts up as $n$ increases, which is readily given by Fig. 2(a), while the coherent motion of the $L+$ mode survives longer as $n$ decreases [26]. In the right panel of Fig. 3, the dynamics of LOPC modes are illustrated with varying the pulse interval $\Delta$ for a fixed carrier density $n=$ $2 \times 10^{18} \mathrm{~cm}^{-3}$. In the figure, the well-defined coherent motion of the $L+$ mode continues up to $\sim 700$ fs at $\Delta=$
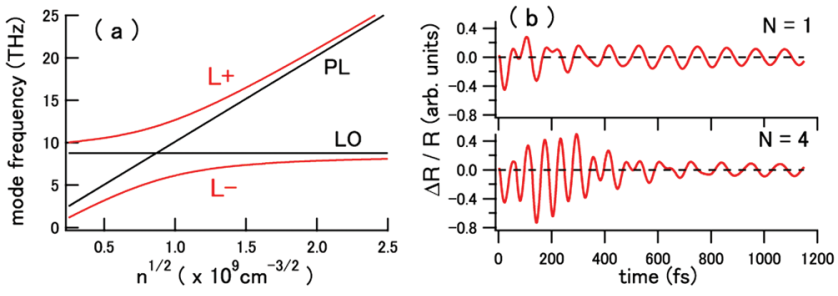

(c)
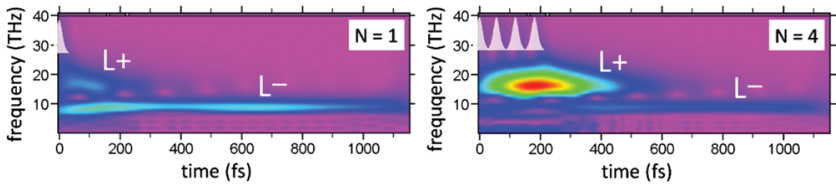

FIG. 2 (color). (a) $L+$ and $L-$ modes (red) for $g=13 \mathrm{meV}$ and bare plasmon and phonon (black). (b) Transient electro-optic responses $\Delta R / R\left[\propto P_{L}(\tau)+P_{E}(\tau)\right.$ with $\left.r_{L} / r_{E}=1\right]$ for the pulse train of $N=1$ and $N=4$. (c) Continuous wavelet transformation of $\Delta R / R$ in the frequency-time space. The density of doped carriers is $n=2\left(\times 10^{18} \mathrm{~cm}^{-3}\right)$ and the interval between pulses is $\Delta=2 \pi / \omega_{L+}$, i.e., $61 \mathrm{fs}$. The detuning $\delta=40 \mathrm{meV}$ is adopted. The applied pulse train is illustrated.
$60 \mathrm{fs}$, but the coherence of the $L+$ mode rapidly disappears at slightly different intervals, i.e., $\Delta=50 \mathrm{fs}$ or $70 \mathrm{fs}$, so that its coherent motion cannot continue that long. It is immediately noted that $\Delta=60 \mathrm{fs}$ is almost the oscillation period of the $L+$ mode, $2 \pi / \omega_{L+}(=61$ fs for $n=2 \times$ $10^{18} \mathrm{~cm}^{-3}$ ). This implies that, in order to control the coherent motion of $L+$ mode, it is essential to synchronize the pulse interval $\Delta$ with the oscillation period of the $L+$ mode. In Fig. 4(a), we provide the behavior of the maximum coherence time $\bar{T}_{L+}$ with respect to $\Delta$ for the $N=8$ pulse train [27], where $\bar{T}_{L+}$ could be the upper bound of $\bar{\tau}_{L+}$. It is clear that $\Delta=2 \pi / \omega_{L+}$ enables us to most efficiently preserve the coherence of the $L+$ mode. It should be noted that $\sim 230 \mathrm{fs}$ is obtained as the lower bound of $\bar{\tau}_{L+}$ at $\Delta=60 \mathrm{fs}$ and $n=2 \times 10^{18} \mathrm{~cm}^{-3}$ by measuring the fast decay after the last pulse of the pulse train in Fig. 3. It enabled the estimation of the lower bound of the carrier mobility of $6038 \mathrm{~cm}^{2} / \mathrm{V} \mathrm{s}$, which is significantly larger than the value of $2074 \mathrm{~cm}^{2} / \mathrm{V} \mathrm{s}$ observed in $n$-GaAs at $300 \mathrm{~K}$ with the similar carrier density of $\sim 2.8 \times$ $10^{18} \mathrm{~cm}^{-3}$ [16].

Finally, returning to the important premise of the present study, we note that it is highly nontrivial to guarantee the virtual electron-hole continuum using the below-band-gap excitation scheme. As a matter of fact, the positive detuning $\left(\delta>0\right.$, i.e., $\left.\omega<\epsilon_{\mathrm{G}}\right)$ is not sufficient to guarantee the virtual excitation because the pulse in principle includes all the frequency components within the broad pulse spectra and also the phase-breaking scattering of virtual excitations can create real ones (finally giving the Urbach tail in the absorption line) [28]. One needs an additional condition regulating the pulse shape or strength. The consideration can be reduced to the dynamics between two levels of the real excited state $\mid r$.ex $\rangle$ and the virtual excited state
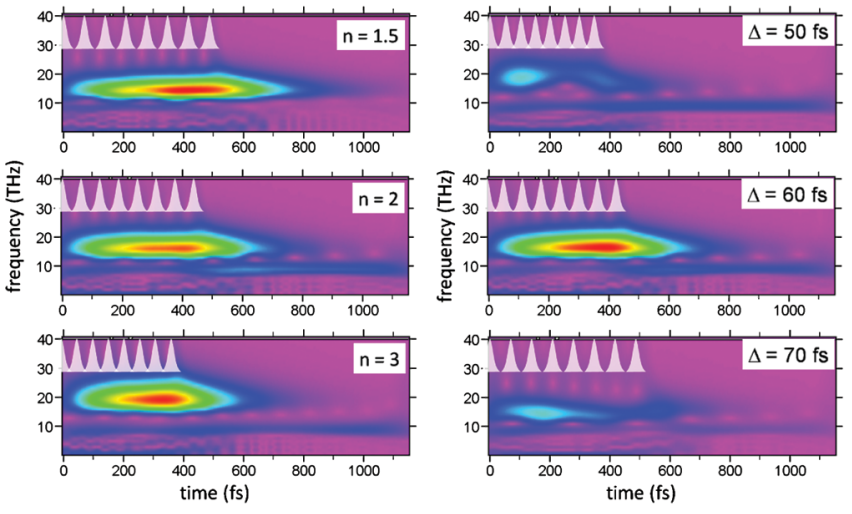

FIG. 3 (color). Continuous wavelet transformation of $\Delta R / R$ in the frequency-time space for the pulse train of $N=8$. Left panel: $\Delta R / R$ with respect to the carrier densities, $n=1.5,2$, and $3\left(\times 10^{18} \mathrm{~cm}^{-3}\right)$ for a fixed pulse interval, $\Delta=2 \pi / \omega_{L+}$. Right panel: $\Delta R / R$ with respect to the intervals between pulses, $\Delta=50,60$, and $70 \mathrm{fs}$ for a fixed density of doped carrier, $n=2$ $\left(\times 10^{18} \mathrm{~cm}^{-3}\right) . \delta=40 \mathrm{meV}$ is taken. The applied pulse train is illustrated in each panel. 

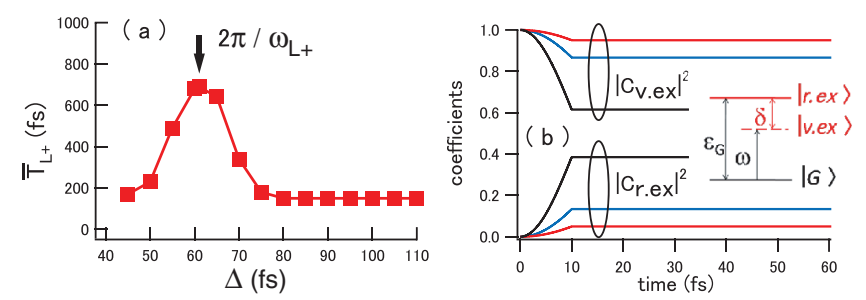

FIG. 4 (color). (a) Behaviors of $\bar{T}_{L+}$ with respect to $\Delta$ for the pulse train of $N=8 . n=2\left(\times 10^{18} \mathrm{~cm}^{-3}\right)$ and $\delta=40 \mathrm{meV}$ are taken. (b) Behaviors of $\left|C_{v \text {.ex }}(\tau)\right|^{2}$ and $\left|C_{\text {r.ex }}(\tau)\right|^{2}$ in the simplified two-level problem for $\Omega_{0}=15 \mathrm{meV}$ (red), $25 \mathrm{meV}$ (blue), and $45 \mathrm{meV}$ (black). $\sigma=10 \mathrm{fs}$ and $\delta=40 \mathrm{meV}$ are adopted. The inset is the level schematic. $|G\rangle$ is the ground state.

$\mid v$.ex $\rangle, \quad$ that $\quad$ is, $\quad i \frac{\partial}{\partial \tau} C_{v \text {.ex }}(\tau)=\Omega(\tau) C_{r \text {.ex }}(\tau) \quad$ and $i \frac{\partial}{\partial \tau} C_{r \text {.ex }}(\tau)=\delta C_{r \text {.ex }}(\tau)+\Omega(\tau) C_{v \text {.ex }}(\tau)$, where $\Omega(\tau)$ depicts the pulse shape, $\Omega(\tau)=\Omega_{0}[\Theta(\tau)-\Theta(\tau-\sigma)]$, and we have $C_{v \text {.ex }}(0)=1$ and $C_{r \text {.ex }}(0)=0$. From Fig. 4(b), it is found that the laser light with the weak field amplitude (say, «15 meV) could be applicable for our purpose. In this weak amplitude limit, it is expected that the scattering event of virtual excitations would be also suppressed. This situation can be also described by the adiabatic Hamiltonian

$$
\mathcal{H}_{0}(\tau)=\left(\begin{array}{cc}
0 & \Omega(\tau) \\
\Omega(\tau) & \delta
\end{array}\right) .
$$

Here we require the condition that the time evolution of $\mid v$.ex $\rangle$ should induce the mixing with $\mid r$.ex $\rangle$ adiabatically, that is, $\mid\langle r . \operatorname{ex} ; \tau|(\partial / \partial \tau \mid v$.ex; $\tau\rangle)|\ll \delta . \quad| v$.ex; $\tau\rangle$ and $|r . \operatorname{ex} ; \tau\rangle$ adiabatically evolve starting from $\mid v$.ex $\rangle$ and $\mid r$.ex $\rangle$, respectively. Keeping only the dominant term, we have $|\dot{\Omega}(\tau) \delta| \ll \delta\left[\delta^{2}+\Omega(\tau)^{2}\right]$. From $\dot{\Omega}(\tau) \approx 2 \Omega_{0} / \sigma$, we finally have $\Omega_{0} \ll \sigma \delta^{2} / 2$. This is in fact the same as the adiabatic condition in the optical control of spin quantum dots [1]. Adopting $\sigma=10 \mathrm{fs}$ and $\delta=40 \mathrm{meV}$, the pulse strength should satisfy $\Omega_{0} \ll 10 \mathrm{meV}$ according to the condition. $\Omega_{0} \rightarrow 0$ in our present study satisfies the required condition well.

To summarize, we have proposed a new theory to control the coherent motion of the LOPC modes and eventually carrier mobility $\mu$ in a polar semiconductor. In the proposition, the dynamics of coherent modes are driven by the virtual electron-hole continuum using the pumping pulse train of below-band-gap excitation, which avoids the dephasing processes and then makes the coherent motion of the carrier-relevant $L+$ mode survive for a very long time under the synchronization of the pulse train with its coherent oscillation. This implies that the carrier mobility $\mu \approx e \bar{\tau}_{L+} / m^{*}$ can be efficiently controlled and dramatically increased, which can be applied also to wide-gap semiconductors such as $\mathrm{GaN}, \mathrm{SiC}, \mathrm{InN}$, and their nanostructures.
We thank Michael Hasselbeck for reading the manuscript. This work was supported by Special Coordination Funds for Promoting Science and Technology and KAKENHI-19540329 from MEXT, Japan.

[1] C. Piermarocchi et al., Phys. Rev. Lett. 89, 167402 (2002).

[2] I. V. Melnikov et al., Phys. Rev. A 56, 1569 (1997).

[3] A. Mysyrowicz et al., Phys. Rev. Lett. 56, 2748 (1986).

[4] S. Schmitt-Rink and D. S. Chemla, Phys. Rev. Lett. 57, 2752 (1986).

[5] T. Unold et al., Phys. Rev. Lett. 92, 157401 (2004).

[6] T. Dekorsy et al., Phys. Rev. B 53, 4005 (1996).

[7] M. C. Beard et al., Phys. Rev. B 62, 15764 (2000).

[8] In a polar semiconductor, an interplay between plasmon and LO phonon results in the new eigenmodes called the LOPC modes: one is the lattice-relevant (i.e., phononlike) $L-$ mode and the other is the carrier-relevant (i.e., plasmonlike) $L+$ mode [9]. There have been extensive studies about the relaxation dynamics of the LOPC modes for GaAs [10,11], InP [12], GaP [13], and InN [14].

[9] A. Mooradian and G. B. Wright, Phys. Rev. Lett. 16, 999 (1966).

[10] G. C. Cho et al., Phys. Rev. Lett. 77, 4062 (1996).

[11] M. Hase et al., Phys. Rev. B 60, 16526 (1999); M. Hase et al., Appl. Phys. Lett. 82, 3668 (2003); H. Altan et al., ibid. 89, 052110 (2006).

[12] R. Huber et al., Phys. Rev. Lett. 94, 027401 (2005).

[13] G. O. Smith et al., Phys. Rev. Lett. 68, 2366 (1992).

[14] Y.-M. Chang et al., Appl. Phys. Lett. 85, 5224 (2004); Y.-M. Chang et al., ibid. 90, 072111 (2007).

[15] S. Nakashima and H. Harima, Phys. Status Solidi A $\mathbf{1 6 2}$, 39 (1997).

[16] M. Hase, Phys. Status Solidi C 5, 364 (2008).

[17] D. C. Langreth, Phys. Rev. B 1, 471 (1970).

[18] B. I. Lundqvist, Phys. Kondens. Mater. 6, 193 (1967).

[19] A. V. Kuznetsov and C. J. Stanton, Phys. Rev. B 51, 7555 (1995).

[20] M. P. Hasselbeck et al., Phys. Rev. B 65, 233203 (2002).

[21] M. I. Bakunov et al., Phys. Rev. B 67, 153201 (2003).

[22] J. D. Lee et al., Phys. Rev. Lett. 97, 157405 (2006).

[23] Phonons and plasmons would relax mainly due, respectively, to anharmonic and carrier-carrier scattering.

[24] In terms of $\mu=e \bar{\tau} / m^{*}, \bar{\tau}=150$ fs corresponds to the carrier mobility of $\sim 8000 \mathrm{~cm}^{2} / \mathrm{Vs}$ in $n$-GaAs with the doping density of $\sim 1 \times 10^{15} \mathrm{~cm}^{-3}$ at $300 \mathrm{~K}$.

[25] B. A. Sanborn, Phys. Rev. B 51, 14256 (1995).

[26] J.D. Lee and M. Hase (unpublished).

[27] Because the temporal profile of $L+$ mode is not relaxational, the definition of $\bar{T}_{L+}$ is rather arbitrary. For Fig. 4(a), $\bar{T}_{L+}$ is defined as the time that the intensity gets less than about $40 \%$ of the maximum intensity at $\Delta=$ $2 \pi / \omega_{L+}$. If the coherent motion of $L+$ mode is suppressed enough, $\bar{\tau}_{\mathrm{PL}}(=150 \mathrm{fs})$ is simply assigned to $\bar{T}_{L+}$.

[28] A. Leitenstorfer et al., Phys. Rev. Lett. 73, 1687 (1994). 